\title{
DYNAMICS OF MULTILAYERED ORTHOTROPIC VISCOELASTIC PLATES OF MAXWELL SOLIDS
}

\author{
P. PAL ROY \\ Blasting Department \\ Central Mining Research Station \\ Barwa Road, Dhanbad 826001 India \\ and

\section{DEBNATH} \\ Department of Mathematics \\ University of Central Florida \\ Or lando, Florida 32816, U.S.A.
}

(Received September 9, 1985 and in revised form January 30, 1987)

\begin{abstract}
This paper is concerned with a simplified dynamical analysis of orthotropic viscoelastic plates that are made up of an arbitrary number of layers each of which is a Maxwell type solid. This study includes the case where some or all the layers are themselves constituted by thinly laminated materials with couple stresses. The recurrence equations for the shear stresses are obtained for an arbitrary number of layers and then applied to plates with two or three layers. The viscoelastic damping effect is determined by the process of linearization and then 1llustrated by a plate composed of one, two or three layers. It is found that the damping increases with anisotropy and wave number. These results are shown by graphical representations.
\end{abstract}

KEYWORDS AND PHRASES. Orthotropic viscoelastic materials, Maxwell solids, Laminated materials and couple stresses.

1980 AMS SUBJECT CLASSIFICATION CODE : $73 \mathrm{~F}$

\section{INTRODUCTION.}

Based upon the general theory of multilayered continua in finite anisotropic elasticity due to Biot [1-4], Pal Roy [5,6] has studied the problems of elastic wave propagation in a thinly layered laminated medium with stress couples under initial stresses. In these studies the composite structures are assumed to be made up of multilayered elastic materials and hence some modifications are required for applications to real solids. It is almost impossible to study statics or dynamics of real solids without suitable approximations and/or assumptions. However, the Maxwell solid is generally believed to be one of the best practical examples of real solids. It is assumed that the Maxwell solid consists of a series of spring with a viscous element known as 'dash pot' (see Biswas [7]), and the main factor which makes this model different from the elastic one is the relaxation time. From a practical point 
of view, the study of statics or dynamics of Maxwell solids seems to be important in its own merit and has physical applications.

In this paper, we study a simplified dynamical analysis of orthotropic viscoelastic plates that are made up of an arbitrary number of layers each of which is a Maxwell type solld. This problem includes the case where some or all the layers are themselves made of thinly laminated materials with couple stresses. The recurrence equations for the shear stresses are derived for an arbitrary number of 1 ayers and then applied to plates with two or three layers. The effect of the viscoelastic damping is examined with examples. The damping is found to increase with anisotropy and wave number. Results are shown by graphical representations.

\section{BASIC EQUATIONS AND ASSUMPTIONS}

We consider a plane strain deformation of a plate of Maxwell solids of thickness $h$. The $x$-axis is chosen midway between the boundaries of the two faces and the $y$-axis is normal to them.

The stress-strain relations for the Maxwe11 solid with the effects of relaxation time, $\tau$ are given by [7]:

$$
\begin{aligned}
& \frac{d}{d t} \sigma_{x x}=2 \mu \frac{d}{d t} e_{x x}-\frac{\sigma_{x x}}{\tau} \\
& \frac{d}{d t} \sigma_{x y}=2 \mu \frac{d}{d t} e_{x y}-\frac{\sigma_{y y}}{\tau} \\
& \frac{d}{d t} \sigma_{y y}=2 \mu \frac{d}{d t} e_{y y}-\frac{\sigma_{y y}}{\tau}
\end{aligned}
$$

where the strain components $e_{1 j}$ are

$$
e_{x x}=\frac{\partial u}{\partial x}, e_{y y}=\frac{\partial v}{\partial y}, e_{x y}=\frac{\partial v}{\partial x}+\frac{\partial u}{\partial y}
$$

The corresponding dynamical equillibrium equations are

$$
\begin{aligned}
& \frac{\partial \sigma_{x x}}{\partial y}+\frac{\partial \sigma_{x y}}{\partial y}=\rho \frac{\partial^{2} u}{\partial t^{2}} \\
& \frac{\partial \sigma_{x y}}{\partial x}+\frac{\partial \sigma_{y y}}{\partial y}=\rho \frac{\partial^{2} v}{\partial t^{2}}
\end{aligned}
$$

where $\sigma_{i j}$ are stress components, $\mu$ is the modulus of rigidity, $\rho$ is the density, $u$ and $v$ are displacement components. The coefficients involved in equations (2.1)

- (2.6) are functions of $y$ as the plate may be inhomogeneous with continuously or discontinuously stratified.

We assume that the displacment fields are harmonic function of time $t$ and sinusoidally distributed along the $x$-direction so that

$$
\begin{aligned}
& u=U(y) \sin m x e^{i \omega t} \\
& v=v \cos m x e^{i \omega t}
\end{aligned}
$$


where $\omega$ is the frequency, $V$ is assumed to be a constant and equal to its average value across the thickness $h$.

From the equation (2.2) we obtain

$$
\sigma_{x y}=M \sin m x A(y) e^{i \omega t}
$$

where $M$ and $A(y)$ are

$$
M=\mu\left(1-\frac{1}{\tau \omega}\right)^{-1} \text { and } A(y)=\frac{d U}{d y}-m V
$$

It follows from $(2.1)$ and (2.7) that

$$
\sigma_{x x}=2 M m U(y) \cos m x e^{i \omega t}
$$

Eliminating $\sigma_{x x}$ and $U$ from (2.11) and (2.5), we obtain

$$
\frac{d}{d y}\left(\frac{1}{N} \frac{d A}{d y}\right)-2 m^{2} A(y)=2 m^{3} V
$$

where

$$
N \equiv N(y)=\frac{1}{2}\left(1-\frac{\rho \omega^{2}}{2 M m^{2}}\right)
$$

The solution for the shearing stress $A(y)$ is determined from equation (2.12) with the boundary conditions $A_{1}$ and $A_{2}$ for $A(y)$ at the top, $y=h / 2$ and the bottom, $y=$ $-\mathrm{h} / 2$ of $:$ he plate. This solution still contains the unknown constant deflection $V$ which is determined by integrating the equilibrium equation (2.6) with respect to y so that

$$
S=-M m \int_{-h / 2}^{h / 2} A(y) d y-m^{2} V \rho_{t}
$$

where the total mass per unit area of the plate face is given by

and

$$
\rho_{t}=\int_{-h / 2}^{h / 2} \rho(y) d y \text {, }
$$

$$
\left[\sigma_{y y}\right]_{1}-\left[\sigma_{y y}\right]_{2}=S \cos m x e^{i \omega t}
$$

represents the total normal load applied to the same unit area. When this normal load is known, the deflection $V$ is obtained from (2.14), and hence the shear stress distribution $A(y)$. Thus $\sigma_{x y}$ is known from (2.9). Once we know $\sigma_{x y}$ the value of $U(y)$ is determined by combining (2.5) to obtain

$$
U(y)=\frac{1}{N m^{2}} \frac{d}{d y} A(y)
$$

Also we obtain $\sigma_{x x}$ from equation (2.11) in the form

$$
\sigma_{x x}=\frac{2 M}{m N} \frac{d}{d y} A(y) \cos m x^{i \omega t}
$$




\section{EXTENSION TO MULTILAYERED PLATES AND VISCOELASTICITY.}

The above results can easily be extended to a multilayered plate constituted by a superposition of thin adherent homogeneous layers each of which is a Maxwell type solid. Within each layer $\mathrm{N}$ is supposed to be a constant. Considering first a single layer of thickness $h$ with $A_{1}$ and $A_{2}$ as the shear stresses at the top and bottom of the layer, it turns out from differential equation (2.12) that

$$
\begin{aligned}
& A=C_{1} \cosh B m y+C_{2} \sinh B m y-m V \\
& B=\sqrt{2 N}=\left(1-\frac{\rho \omega^{2}}{2 m^{2}}\right)^{\frac{1}{2}}, \\
& C_{1}=\left[\frac{1}{2}\left(A_{1}+A_{2}\right)+m V\right] \frac{1}{\cosh B \gamma} \\
& C_{2}=\frac{1}{2}\left(A_{1}-A_{2}\right) \frac{1}{\sinh B \gamma} \\
& \gamma=(m h / 2), M=\mu\left(1-\frac{1}{\omega \tau}\right)^{-1}
\end{aligned}
$$

The displacement amplitude $U$ is given by equation (2.17). Its values $U_{1}$ and $U_{2}$ at the top and bottom of the layer are found to be

$$
\begin{aligned}
& \mathrm{U}_{1}=\frac{1}{\sqrt{2 \mathrm{~N}} \mathrm{~m}}\left(\mathrm{~A}_{1} \mathrm{a}+\mathrm{A}_{2} \mathrm{~b}\right)+2 \mathrm{Vc} \\
& \mathrm{U}_{2}=-\frac{1}{\sqrt{2 \mathrm{~N}} \mathrm{~m}}\left(\mathrm{~A}_{1} \mathrm{~b}+\mathrm{A}_{2} \mathrm{a}\right)-2 \mathrm{Vc}
\end{aligned}
$$

with $a=\tanh B \gamma+\frac{1}{\tanh B \gamma}, \quad b=\tanh \beta \gamma-\frac{1}{\tanh \beta \gamma}, \quad c=\frac{1}{\beta} \tanh \beta \gamma \quad$ (3.8abc)

We consider now a plate composed of $n$ orthotropic homogeneous layers. The jth layer $(j=1,2 \ldots n)$ of thickness $h_{j}$ is characterized by coefficients $\mu_{j}$, $\tau_{j}$ and a mass density $\rho_{j}$ with the corresponding parameters $a_{j}, b_{j}, c_{j}, M_{j}, N_{j}$. The displacement amplitude and shear stress at the top and bottom of the $j$ th layers are respectively $\left(U_{j}, A_{j}\right)$ and $\left(U_{j+1}, A_{j+1}\right)$.

Now, at the interface between the layers $j$ and $(j+1)$ the displacement amplitude $U_{j+1}$ as it is found for the lower face of the $j$ th layer and upper face of the $(j+1) t h$ layer, must be the same. We obtain from equations (3.6) - (3.7)

where

$$
A_{j} D_{j}+\left(E_{j}+E_{j+1}\right) A_{j+1}+A_{j+2} D_{j+1}=-2 V\left(c_{j}+c_{j+1}\right)
$$

$$
E_{j}=\frac{a_{j}}{\sqrt{2 N} m}, D_{j}=\frac{b_{j}}{\sqrt{2 N} m}
$$

Thus when the values of the shear stresses $A_{1}$ and $A_{n+1}$ are given at the outer boundaries, the recurrence equations (3.9) will lead to the evaulatuion of the ( $n-1)$ shear stresses $A_{j}(j=1,3 \ldots n)$ at the interfaces.

The normal load $S_{f}$ acting on the $j t h$ layer is obtained from equations (2.14) and (3.1) and has the form 


$$
s_{j}=-M_{j} c_{j}\left(A_{j}+A_{j+1}\right)+m^{2} V h_{j} M_{j}\left(1-\frac{c}{r_{j}}\right)-m^{2} \rho_{j} V h_{j}
$$

The total load $S$ applied to the multi-layered plate is obtained by summing the loads $\mathrm{S}_{j}$ applied to each layer. We obtain

$$
S=\sum_{j=1}^{n} s_{j}=-\sum_{j} M_{j} c_{j}\left(A_{j}+A_{j+1}\right)+m^{2} v k-m^{2} o_{t} v
$$

with

$$
\rho_{t}=\Sigma_{j} \rho_{j} h_{j}, \quad k=\Sigma_{j} h_{j} M_{j}\left(1-\frac{c}{\gamma_{j}}\right), \quad \gamma_{j}=\left(m h_{j}\right) / 2
$$

The equation (3.12) will provide the only unknown $V$ of the system. When this unknown $V$ is known, the shear stresses $A_{j}$ at the interfaces are also completely known.

\section{VISCOELASTIC MATERIALS.}

We next consider the viscoelastic properties of the layers for possible technological applications of the above analysis. It follows from (2.4a) and (2.7) that the strain component is

$$
e_{x x}=m U(y) \cos m x e^{i \omega t}
$$

Hence we find from (2.11) that

$$
\sigma_{x x}=2 M e_{x x}
$$

Also it follows from $(2.4 \mathrm{c})$ with $(2.7)-(2.9)$ that

$$
\sigma_{x y}=2 M e_{x y}
$$

It is clear from equations (4.2) and (4.3) that for Maxwell type solid there is only one coefficient $\hat{M}$ instead of two coefficients for the elastic materials. Viscoelastic properties of the layers may be taken into account by substituting the following general form of operator $\hat{M}$ for the coefficient $M$ (see Biot [8]):

$$
\hat{M}=\int_{0}^{\alpha} \frac{p}{p+r} M(r) d r+M^{\prime \prime}+M^{\prime} p
$$

where $p=\frac{d}{d t}$. For harmonic oscillations $p=i \omega$ and the operator also becomes a complex quantity.

\section{THINLY LAYERED LAMINATED MATERIALS.}

We now consider a multi-layered plate in which the layers are themselves composed of thinly laninated materials of Maxwell solids. For a laminated medium composed of a repeated sequence of $n$ thin layers each of which occupies a fraction $\delta_{j}(j=1,2 \ldots n)$ of the total thickness $h^{\prime}$ of the laminated medium and characterized by the coefficient $M_{j}=\mu_{j} /\left(1-\frac{i}{\omega \tau}\right)$. The equivalent coefficient $M$ i.e. the coefficient of the equivalent anisotropic meddium) is 


$$
M=\sum_{j=1}^{n} M_{j} \delta_{j}
$$

This equivalent coefficient $M$ constitute a first approximation. The next approximation is provided by introducing stress couples [3] i.e. a moment per unit area equal to

$$
R=b \frac{\partial^{2} v}{\partial x^{2}}
$$

where $\quad b=\frac{\left(h^{\prime}\right)^{2}}{3} \sum_{j=1}^{n} M_{j} \delta_{j}^{3}\left(1-\frac{M}{M_{j}}\right)$

The equilibrium equations (2.5) - (2.6) are now modified to the following form

$$
\begin{aligned}
& \frac{\partial}{\partial x} \sigma_{x x}+\frac{\partial}{\partial y} \sigma_{x y}=\rho \frac{\partial^{2} u}{\partial t^{2}} \\
& \frac{\partial}{\partial x} \sigma_{x y}+\frac{\partial}{\partial y} \sigma_{y y}=\rho \frac{\partial^{2} v}{\partial t^{2}}+b \frac{\partial^{4} v}{\partial x^{4}}
\end{aligned}
$$

Consequently, equation ( 3.12$)$ must be replaced by

$$
S=-\sum_{j=1}^{n}\left(A_{j}+A_{j+1}\right) c_{j} M_{j}+m^{2} V k-\omega^{2} \rho_{t} \hat{v}+m^{4} v \sum_{j=1}^{n} b_{j} h_{j}
$$

where $b_{j}(j=1,2 \ldots n)$ is the couple stress coefficient of the $j t h$ laminated medium. The result (5.6) can immediately be extended to viscoelastic laminated media.

6. EVALUATION OF DAMPING.

An important task in the problem of design analysis is the determination of the effect of viscoelastic layers on vibration absorption at resonance. We consider a simply supported homogeneous anisotropic plate. The span $p$ equal to half the wave length is

$$
p=\frac{\pi}{m}
$$

From equations (2.14) and (3.1) we have the expression for $S$ which, after putting $A_{1}=0$ and $A_{2}=0$, becomes

$$
S=m^{2} h M V\left(1-\frac{\tanh B \gamma}{B \gamma}\right)-m^{2} \rho_{t} V
$$

where $\quad B=\sqrt{2 N}=\left(1-\frac{\rho \omega^{2}}{2 M m^{2}}\right)^{\frac{1}{2}}$

For a viscoelastic material $M$ is replaced by $M+\bar{M}$ where $\bar{M}$ is the purely imaginary term. The imaginary part of the load $S$ is represented by $\bar{S}$ and 18 obtained from equation $(6.2)$ by linearizing it with respect to $\bar{M}$. We obtain

$$
\bar{S}=m^{2} \operatorname{Vh} F \bar{M}
$$


with $F=1-\frac{3}{2} \frac{\tanh \beta \gamma}{\beta \gamma}+\frac{1}{2} \frac{1}{\cosh ^{2} \beta \gamma}+\frac{1}{2 \beta^{2}}\left(\frac{\tanh \beta \gamma}{\beta \gamma}-\frac{1}{\cosh ^{2} \beta}\right)$

Equation (6.3) determines the damping (longitudina1) on vibration attenuation.

The variation of $F$ for different values of $\beta$ (anisotropy) and $\gamma$ are shown in figure 1. It is seen that the damping increases with anisotropy and wave number. The result (6.3) is also applicable to the laminated plate of the equivalent continum as described in section 5. In the case the inclusion of couple stress will contribute an additional term $\mathrm{m}^{4} \mathrm{Vh} \Delta \mathrm{b}$ where $\Delta \mathrm{b}$ is the imaginary part of equation (5.3).

From equation (2.10a) and (6.3) it follows that

$$
\overline{\mathrm{s}}=\mathrm{p} \cdot \mathrm{F}
$$

with

$$
p=\frac{m^{2} V h \mu \omega \tau}{1+\omega^{2} \tau^{2}}
$$

The variations of $\frac{p}{\mu}$ with $\omega \tau$ are shown in Figure 2 which confirms the symmetric variation with respect to the relaxation time $\tau$. As a particular example, we consider a plate composed of two layers. The material (obviously Maxwell type) of the first layer of thickness $h^{\prime}$ is characterized by the coefficient $M_{1}$ and that the second layer of thickness $h^{\prime \prime}$ is characterized by the coefficient $M_{2}$ and suppose $h=$ $h^{\prime}+h^{\prime \prime}$.

The applied shear stresses at the outerfaces are put equal to zero (1.e. $A_{1}=A_{3}=0$. The shear stress $A_{2}$ at the interface is found by equating the interfacial displacement amplitude $U_{2}$ consldered as belonging to the first and second layer. Applying equations (3.3) we obtain

$$
\begin{aligned}
& \mathrm{U}_{1}=-\frac{1}{\sqrt{2 \mathrm{~N}_{1} m}\left(A_{2} a_{1}\right)-2 \mathrm{~V}_{2}} \\
& \mathrm{U}_{2}=\frac{1}{\sqrt{2 \mathrm{~N}_{2} \mathrm{~m}}\left(\mathrm{~A}_{2} \mathrm{a}_{2}\right)+2 \mathrm{~V} c_{1}}
\end{aligned}
$$

where $N_{1}$ and $N_{2}$ are the values of $N$ for $M=M_{1}$ and $M=M_{2}$ respectively. The total load $q\left(=q_{1}+q_{2}\right)$ is given by

$$
\frac{q}{V m}=\frac{2\left(M_{1} c_{1}+M_{2} c_{2}\right)\left(c_{1}+c_{2}\right)}{\left(\frac{a_{1}}{\sqrt{2 N_{1}}}+\frac{a_{2}}{\sqrt{2 N_{2}}}\right)}+m\left(h^{\prime} M_{1}+h^{\prime \prime} \cdot M_{2}\right)-2\left(M_{1} c_{1}+M_{2} c_{2}\right)-\frac{g^{2}}{m}\left(\rho_{1} h^{\prime}+\rho_{2} h^{\prime \prime}\right)
$$

This equation determines the deflection $V$ when the normal load applied to the structure is given.

We next consider a plate composed of three layers. The first and third layers are identical in nature and are characterized by the coefficient $M_{1}$ while the middle one is characterized by the coefficient $M_{2}$. Because of the symmetry, the interfacial shears are $A_{2}=A_{3}$ while $A_{1}=A_{4}=0$ at the outerfaces. If we equate the interfacial displacements $\mathrm{U}_{2}$ considered as belonging to layers 1 and 2 . We obtain 


$$
A_{2}=-\frac{2\left(c_{1}+c_{2}\right) m V}{\left(\frac{a_{1}}{\left.\sqrt{2 N_{1}}+\frac{2 \mathrm{~B}_{2} c_{2}}{\sqrt{2 N_{2}}}\right)}\right.}
$$

where $B_{2}$ is given by $a_{2}+b_{2}=2 B_{2} c_{2}$.

The total load $q=q_{1}+q_{2}$ in this case is given by

$$
\frac{q}{V_{m}}=\frac{2\left(c_{1}+c_{2}\right)\left(2 M_{1} c_{1}+M_{2} c_{1}\right.}{\left(\frac{a_{1}}{\sqrt{2 N}}+\frac{2 N_{1} c_{2}}{\sqrt{2 N_{2}}}\right)}+m\left(2 h_{1} M_{1}+h_{2} M_{2}\right)-2\left(2 M_{1} c_{1}+M_{2} c_{2}\right)-\frac{\omega^{2}}{m}\left(2 \rho_{1} h^{\prime}+\rho_{2} h^{\prime \prime}\right)
$$

The second and third terms represent the normal load corresponding to superposed layers with perfect interfacial slip while the first term represents the effect of adherence between layers.

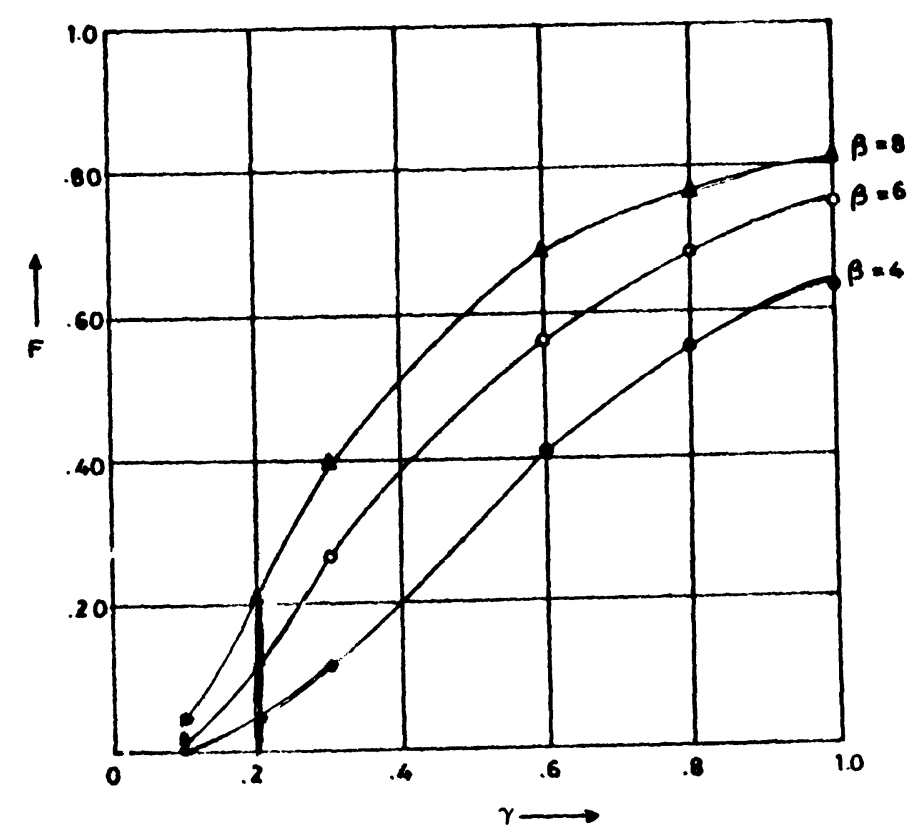

Fig. 1 Variation of $F$ with $\gamma$ for different values of $\beta$ (equation 6.3). 


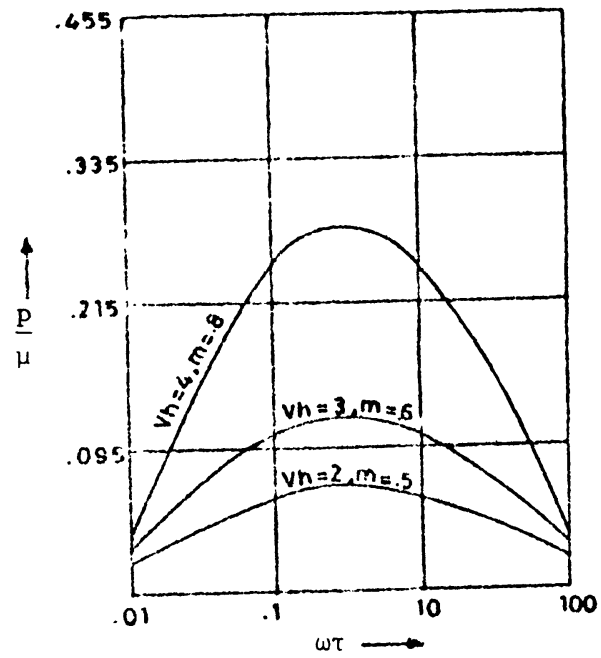

Fig. 2 Variation of $\frac{p}{\mu}$ over $\omega \tau$.

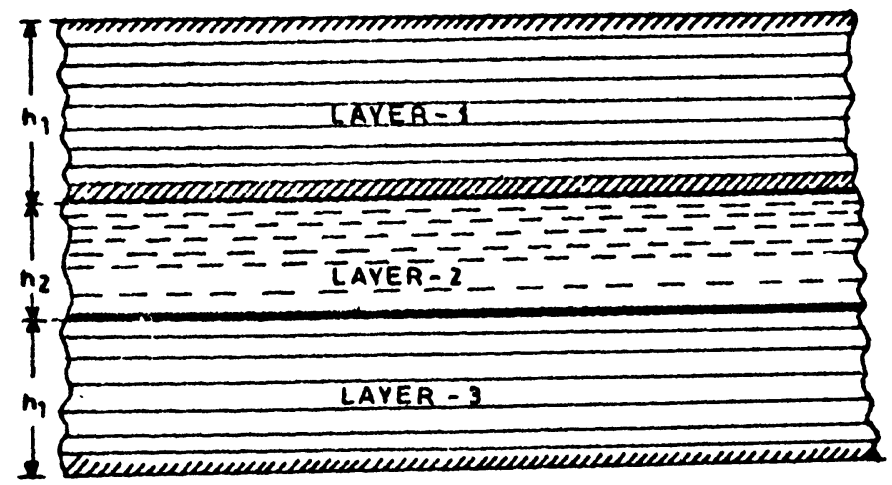

Fig. 3 A three-layered symmetric plates composed of a core of thickness $h_{2}$ sandwiched between two identical layers of thickness $h_{1}$.

7. CONCLUDING REMARKS.

The foregoing results of a simply supported plate with a loading distributed as a half sine wave are obviously valid for an arbitrary loading provided it can be expanded in a series along the span. In this case, the results are applied to the various Fourier components with a suitable value of $m$ corresponding to each wave length. The procedures used for the simply supported case can easily be extended to the case where the plate is built-in (i.e. when both end points of the plate are rigidly attached so that the displacement of its faces are both zero. In this case, in order to satisfy the boundary conditions we must, instead of sinusoidal solutions, consider exponential solutions. Such solutions are empirically derived from the trigonometric solutions $(2.7)$ and $(2.8)$ if we replace $m$ by ik. 
ACKNOWLEDGEMENT. The research of the first author was partially supported by the UGCDSA Program of Jadavpur University, Calcutta, India. This work was also partially supported by the University of Central Florida.

\section{REFERENCE $S$}

1. BIOT, M.A. Mechannics of Incremental Deformations, John Wiley and Sons, New York (1965).

2. BIOT, M.A. Theory of Stability of Multilayered Continua in Finite Anisotropic Elasticity, J. Franklin Inst. 276 (1963), 128-153.

3. BIOT, M.A. A New Approach to the Mechanics of Orthotropic Multilayered Plates, Int. J. Solids Struct. 8 (1972), 475-490.

4. BIOT, M.A. Simplified Dynamics of Multilayered Orthotropic Viscoelastic P1ates, Int. J. Solids Struct. 8 (1972), 491-509.

5. PAL ROY, P. A Simplified Approach to the Propagation of Edge Waves in a Thinly Layered Laminated plate with Stress Couples Under Biaxial Initial Stresses, Acta Geoph. Pol. 33 No. 3 (1985), 329-339.

6. PAL ROY, P. Wave Propagation in a Thinly Two-Layered Laminated Medium with Stress Couples Under Initial Stresses, Acta Mech. 54 (1984), 1-21.

7. BISWAS, A. Transmissions of Love Type Waves Through a Maxwell Solid Layer Sandwich in Homogeneous Elastic Media, Pure and Appl. Geoph. 69 $(1968) / I), 75-79$.

8. BIOT, M.A. Theory of Stress-Strain Relations in Anisotropic Viscoelastic and Relaxation Phenomena, J. App. Phys. 25 (1954), 1385-1391. 


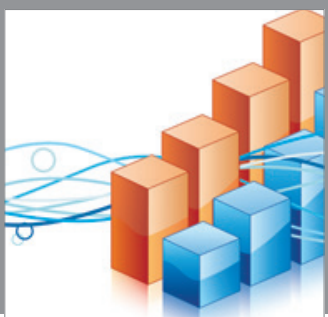

Advances in

Operations Research

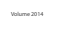

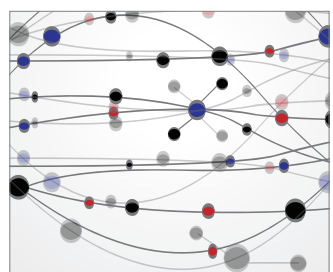

\section{The Scientific} World Journal
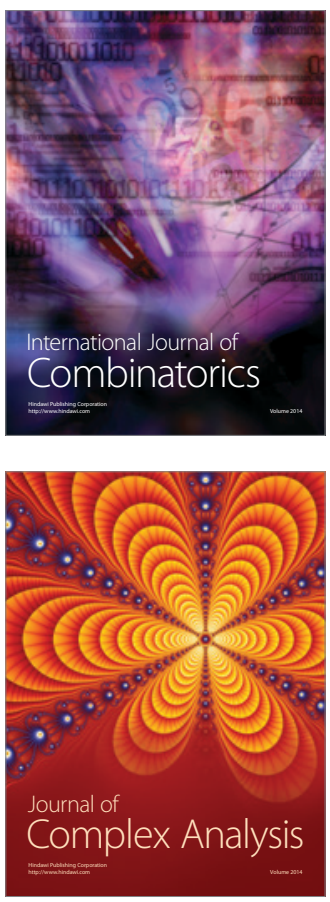

International Journal of

Mathematics and

Mathematical

Sciences
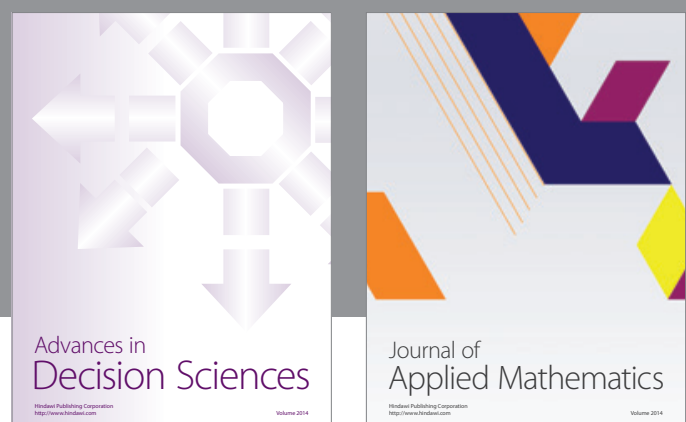

Journal of

Applied Mathematics
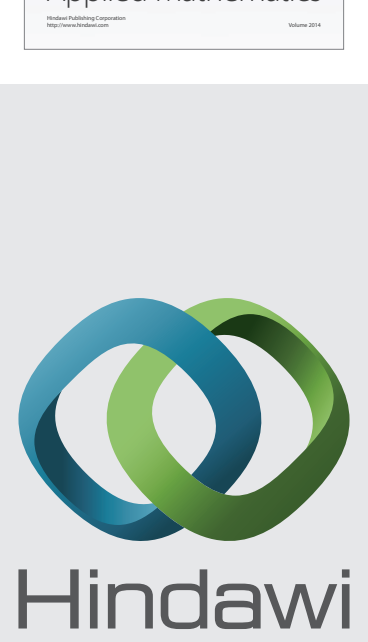

Submit your manuscripts at http://www.hindawi.com
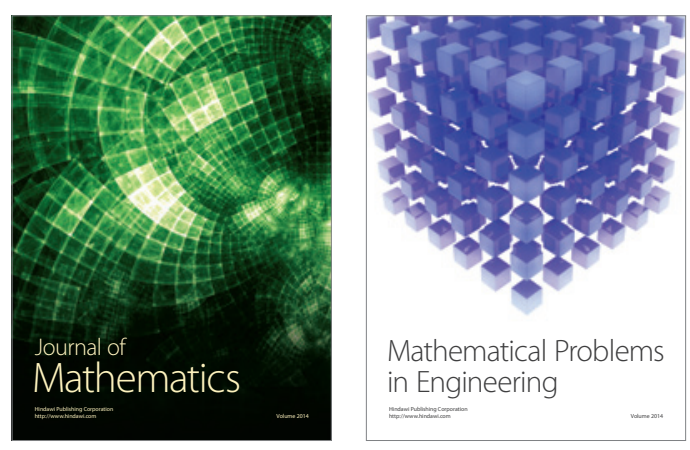

Mathematical Problems in Engineering
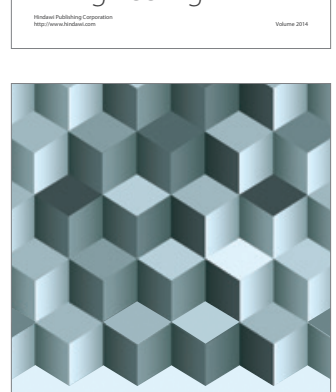

Journal of

Function Spaces
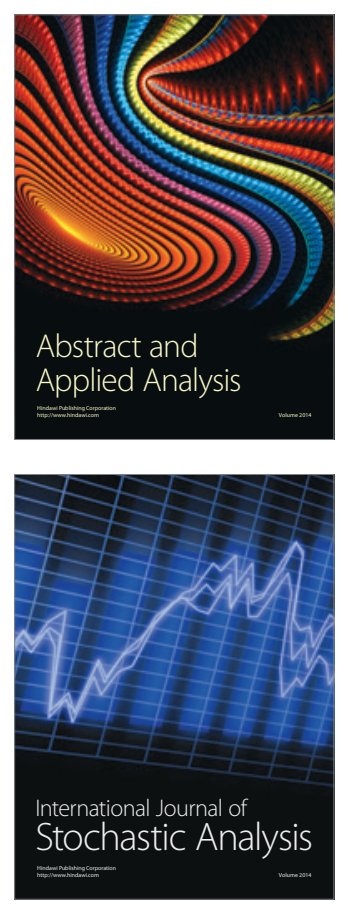

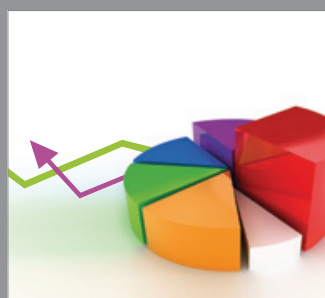

ournal of

Probability and Statistics

Promensencen
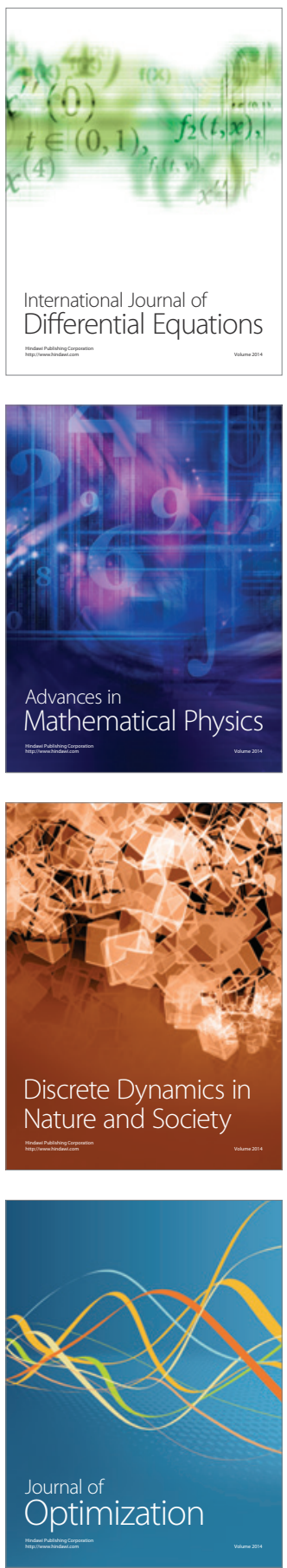